\title{
Análise de transientes hidráulicos e de chaminés de equilíbrio em sistemas de bombeamento de água por meio de otimização das vazões e dos volumes de reservação \\ Analysis of hydraulic transients and standpipes in water pumping systems by optimizing flows and reservoir volumes \\ Data de entrada: $17 / 03 / 2020$ \\ Data de aprovação: 01/10/2020
}

Raynner Menezes Lopes ${ }^{1 *}$ | Marcelo Giulian Marques ${ }^{2}$ | Murício Dai Prá | José Almir Rodrigues Pereira ${ }^{3}$

DOI: https://doi.org/10.36659/dae.2022.022

\section{ORCID ID}

Lopes RM (iD) https://orcid.org/0000-0001-8346-1280

Marques MG (1D) https://orcid.org/0000-0002-9948-3094
Prá MD (D) https://orcid.org/0000-0002-1858-1531

Pereira JAR (1D) https://orcid.org/0000-0002-3144-1787

\section{Resumo}

Neste estudo, foram aplicados dois métodos analíticos de estimativa de transientes hidráulicos a 105 Sistemas de Bombeamento de Água (SBAs) fictícios. Esses métodos foram o de Mendiluce e o de Tassinari (2017). Também foram comparados dois métodos de verificação da necessidade de chaminé de equilíbrio, no caso, o adimensional $\mathrm{L} / \mathrm{Hm}$ e o tempo de desaceleração do escoamento (th). O volume da chaminé foi calculado. As vazões de bombeamento (Q⿱b) e volume do reservatório elevado (VRE) foram otimizados por algoritmo genético evolutivo. Nos resultados, o método de Mendiluce foi recomendado para SBAs com comprimento (L) de até $500 \mathrm{~m}$. O de Tassinari (2017) pode ser utilizando com o primeiro método em SBAs de L entre $500 \mathrm{~m}$ e $1000 \mathrm{~m}$. O adimensional L/Hm foi o método com maior segurança na verificação de necessidade de chaminé. Foram ajustadas equações explícitas para que o projetista obtenha de forma prática Qb e VRE com menor custo global para o SBA.

Palavras-chave: Golpe de aríete. Algoritmo genético evolutivo. SCE-UA.

\section{Abstract}

In this study, two analytical methods of estimating hydraulic transients, one proposed by Mendiluce (2017) and another by Tassinari (2017), were applied to 105 fictitious Water Pumping Systems (WPSs). Two methods of assessing the need for an standpipe were also compared, namely the adimensional $\mathrm{L} / \mathrm{Hm}$ and the flow deceleration time (th). The standpipe volume was calculated. The pumping flow rates (Qb) and the high reservoir volume (HRV) were optimized by evolutionary genetic algorithm. In the results, the Mendiluce method was recommended for WPSs with length (L) up to $500 \mathrm{~m}$, while that of Tassinari (2017) can be used with the first method in WPSs of L between

\footnotetext{
${ }^{1}$ Universidade Federal do Pará - Tucuruí - Pará - Brasil.

${ }^{2}$ Universidade Federal do Rio Grande do Sul - Porto Alegre - Rio Grande do Sul - Brasil.

${ }^{3}$ Universidade Federal do Pará - Belém - Pará - Brasil.

* Autor correspondente: raynnerlopesaufpa.br.
} 
$500 \mathrm{~m}$ and $1000 \mathrm{~m}$. The adimensional $\mathrm{L} / \mathrm{Hm}$ was the safest method to check the need for a standpipe. Explicit equations were adjusted so that the designer obtains $Q b$ and $H R V$ in a practical manner with lower overall costs for the WPS.

Keywords: Water hammer. Evolutionary genetic algorithm. SCE-UA.

\section{INTRODUÇÃO}

A operação de um Sistema de Bombeamento de Água (SBA), especialmente aquele comvelocidade rotacional constante, é caracterizada pela intensidade das pressões extremas que ocorrem principalmente durante a interrupção do regime permanente de bombeamento, isto é, durante o desligamento do Conjunto Motor e Bomba (CMB). Essas pressões extremas, denominadas de transientes hidráulicos, transitórios hidráulicos ou golpes de aríete, podem deformar (KERAMAT et al., 2012) e colapsar a linha de adução, causando prejuízos às concessionárias de abastecimento de água.

Apesar de não ser fácil estimar os transientes hidráulicos em um SBA, esse procedimento é essencial aos engenheiros projetistas e construtores por garantir a eficiência econômica global e a segurança nas operações das concessionárias (TRIKI, 2016). Por isso, muitos pesquisadores estudaram o fenômeno do golpe de aríete com diferentes abordagens nas últimas décadas (GAD e MOHAMMED, 2014). A NBR 12215-1/2017, da Associação Brasileira de Normas Técnicas (ABNT), recomenda que essa atividade seja realizada com software específico, cuja modelagem matemática se aproxime do sistema hidráulico em estudo e/ou projeto. Também é recomendado que o software tenha métodos numéricos, como o método das características, métodos das ondas planas, método de diferenças finitas e o método de elementos finitos; e que resolvam as equações de derivadas parciais governantes dos fenômenos transitórios considerando as características físicas da água e das tubulações e dos vínculos estruturais das tubulações com o meio externo (ABNT, 2017).
Mesmo com a complexidade do estudo dos transientes hidráulicos, o que só pode ser feito mediante a utilização de certa quantidade de dados de entrada, na NBR 12215-1/2017 da ABNT é enfatizado que a análise de transitórios deve ser efetuada em diferentes etapas do estudo e/ou projeto, como no estudo de concepção, no projeto básico e no projeto executivo.

Na etapa inicial de concepção do SBA, em que ainda são poucas as informações do sistema, os métodos simplificados de estimativa dos transientes são recurso conveniente e importante para o engenheiro projetista, pois são métodos que não necessitam de recurso computacional e podem ser calculados com mais facilidade, pois geram resultados explícitos utilizando número menor de dados de entrada.

É importante destacar que os métodos simplificados não devem substituir os métodos numéricos; no entanto, auxiliam o projetista nas previsões iniciais dos transientes no SBA, contribuindo com a tomada de decisão da melhor alterativa de concepção técnica do sistema.

Tomando como base os conceitos de praticidade e objetividade que devem fazer parte do cotidiano do engenheiro, a finalidade é o estudo das aplicações do método de estimativa de transientes hidráulicos proposto por Tassinari (2017), em conjunto com o método clássico de Mendiluce, bem como o estudo de métodos de verificação da necessidade de dispositivo de proteção contra transientes em um SBA. Também foi estudada a influência do diâmetro da linha de recalque do SBA na vazão de bombeamento (Q⿱ b), no volume do reservatório elevado (VRE) e no volume do dispositivo de proteção contra o transiente. 


\section{METODOLOGIA}

$\mathrm{Na}$ realização do estudo foram selecionados e combinados diversos valores das variáveis que caracterizam um SBA, sendo elas a altura geométrica $(\mathrm{Hg})$, o comprimento $(\mathrm{L})$, o diâmetro interno (Di) e o material da linha de adução.
$\mathrm{Na}$ Tabela 1 são apresentadas as características selecionadas para a tubulação. 0 material adotado foi o Ferro Fundido $\left(\mathrm{F}^{\circ} \mathrm{F}^{\circ}\right)$, sendo analisadas 105 configurações de SBAs (5 valores de $\mathrm{Hg} \times 3$ valores de $L \times 7$ valores de $\mathrm{Di}$ ). Um exemplo de um dos 105 sistemas analisados é com $\mathrm{Hg}=10 \mathrm{~m}$, $\mathrm{L}=500 \mathrm{~m}$ e $\mathrm{Di}=480 \mathrm{~mm}$.

Tabela 1 - Valores de $\mathrm{Hg}$ e L e Di ${ }^{1}$ utilizados no estudo.

\begin{tabular}{|c|c|c|c|c|c|c|c|}
\hline $\mathbf{H g}(\mathbf{m})$ & 10 & 25 & 50 & 75 & 100 & - \\
\hline $\mathbf{L}(\mathbf{m})$ & 100 & 500 & 1000 & - & - \\
\hline $\mathrm{Di}(\mathbf{m m})$ & 274 & 326 & 378 & 429 & 480 & - \\
\hline
\end{tabular}

Nos 105 SBAs foram determinados regimes de escoamento por meio da otimização de Qb. Para a otimização, foi criada a função objetivo, a partir de outras equações, como a equação da perda de carga hidráulica de Hazen-Williams, para determinar a altura manométrica $(\mathrm{Hm})$ do sistema, a equação da potência $(P)$ requerida pelo sistema para recalcar $\mathrm{Qb}$, as equações de custo do $\mathrm{kWh}$ bombeado, as equações de custo de implantação do SBA e o volume da chaminé de equilíbrio (Vc). Qb e VRE foram utilizados como variáveis de decisão da função objetivo, que é a soma do custo de operação com o custo de implantação do sistema. Para isso, foi utilizado o algoritmo de otimização Shuffled Complex Evolution - University of Arizona (SCE-UA), com a finalidade de minimizar a função objetivo. Os intervalos de busca, isto é, os limites inferiores e superiores para Qb de cada um dos 105 sistemas, foram aqueles que resultaram em velocidade de $0,3 \mathrm{~m} / \mathrm{s}$ e $3 \mathrm{~m} / \mathrm{s}$, respectivamente, atendendo ao recomendado na NBR 12215-1/2017 da ABNT para o bombeamento de água tratada. Os limites inferior e superior para VRE foram de $500 \mathrm{~m}^{3}$ e $1000 \mathrm{~m}^{3}$, respectivamente. Assim, com o algoritmo SCE-UA buscaram-se, para cada SBA fictício, os valores de Q Q e
VRE otimizados, que são os valores para os quais o sistema terá menor custo de operação e menor custo de implantação.

Os regimes de escoamento para os valores de $Q b$ otimizados em cada SBA foram utilizados para verificar a necessidade de chaminé de equilíbrio, calcular o seu volume e estimar os transientes hidráulicos. Os transientes foram determinados por dois métodos analíticos. O primeiro foi o método de Mendiluce, que é clássico e está a favor da segurança (LOPES et al., 2018), como segundo método foi utilizado o proposto por Tassinari (2017), que necessita de mais aplicações.

Considerando a impossibilidade de exibir todas as equações em detalhes, a seguir são descritos a metodologia de cálculo dos transientes e o cálculo do volume da chaminé equilíbrio.

\subsection{Métodos de Análise de Transientes Hidráulicos}

O método de Mendiluce foi aplicado com a utilização da Eq. de 1 a 6. Para o cálculo da celeridade $a(\mathrm{em} \mathrm{m} / \mathrm{s})$ da onda foi utilizada a Eq 1.

\footnotetext{
${ }^{1}$ Os valores de Di foram retirados de um catálogo de um conhecido fabricante de tubos para linhas de condução de água.
} 


$$
a=\frac{9900}{\sqrt{48,3+\mathrm{k} \cdot \frac{\mathrm{Di}}{e}}}
$$

Onde $\mathrm{k}$ (adimensional) é coeficiente que considera os módulos de elasticidade do tubo, Di (em $\mathrm{mm}$ ) é o diâmetro interno do conduto e $e$ é a espessura (em $\mathrm{mm}$ ) do tubo.

O cálculo do período $\tau$ (em s) da tubulação foi obtido com a Eq. 2.

$\tau=\frac{2 . \mathrm{L}}{a}$

Onde L é o comprimento da tubulação (em m). Em seguida, com a Eq. 3 foi obtido o tempo de parada tp (em s).

$$
t p=\mathrm{C}+K_{i} \cdot t h
$$

Onde C (adimensional) é coeficiente obtido por meio de experimentos, em função do parâmetro adimensional $\mathrm{Hm} / \mathrm{L}$, o coeficiente (adimensional) Ki representa o efeito da inercia das partes girantes do CMB e th é o tempo de desaceleração do escoamento (em s), que foi obtido pela Eq.4.

$$
\text { th }=\frac{\mathrm{vL}}{\mathrm{gH}_{\mathrm{m}}}
$$

Onde vé a velocidade de escoamento (em $\mathrm{m} / \mathrm{s}), \mathrm{g}$ é a aceleração da gravidade $\left(\mathrm{em} \mathrm{m} / \mathrm{s}^{2}\right)$

Para o caso de $t p<\tau$, a manobra é classificada rápida, e a equação a ser utilizada para o cálculo da sobre pressão $(+\Delta H)$ e sub pressão $(-\Delta H)$, em m.c.a, é a Eq. 5.
$\Delta \mathrm{H} \pm=\frac{\mathrm{a} \cdot \mathrm{v}}{\mathrm{g}}$

Para o caso de tp $\geq \tau$, a manobra é classificada como lenta, e a Eq. 6. deve ser utilizada.

$\Delta \mathrm{H} \pm=\frac{2 . \mathrm{L} \cdot \mathrm{v}}{\text { g.tp }}$

No método proposto por Tassinari (2017) existe um conjunto de equações ajustadas a partir do software Allievi ${ }^{2}$, que permite determinar envoltórias de máximos e mínimos em três pontos distintos da linha de adução. $O$ primeiro ponto é próximo ao conjunto elevatório (a 0\% de L), o segundo a $50 \%$ de L e o terceiro a 75\% de L. A Eq. 7 e Eq. 8 são utilizadas para determinar as sobre pressões $(\mathrm{H}+)$ e sub pressões $(\mathrm{H}-$ ), respectivamente, a $0 \%$ de $\mathrm{L}$ (junto ao CMB). Com a Eq. 9 e a Eq. 10 são determinados os transientes a $50 \%$ de $L$, enquanto com a Eq. 11 e a Eq. 12 são determinados a $75 \%$ de L:

$$
\begin{aligned}
& \mathrm{H}+=-0,0183 \cdot \mathrm{th}^{2}+0,1484 \cdot t h+0,4261 \\
& \mathrm{H}-=-0,0064 \cdot \mathrm{th}^{3}+0,0864 \cdot \mathrm{th}^{2}-0,3686 \cdot \mathrm{th}-0,3473 \\
& \mathrm{H}+=-0,0160 \cdot \mathrm{th}^{2}+0,1207 \cdot \mathrm{th}+0,2886 \\
& \mathrm{H}=-0,0061 \cdot \mathrm{th}^{3}+0,0784 \cdot \mathrm{th}^{2}-0,3279 \cdot \mathrm{th}-0,2066 \\
& \mathrm{H}+=-0,0145 \cdot \mathrm{th}^{2}+0,1213 \cdot \mathrm{th}+0,146 \\
& \mathrm{H}=-0,0037 \cdot \mathrm{th}^{3}+0,0494 \cdot \mathrm{th}^{2}-0,2283 \cdot \mathrm{th}-0,1083
\end{aligned}
$$

Onde th é o tempo de desaceleração do escoamento (em s).

O método de Tassinari (2017) pode ser aplicado para tubos de $\mathrm{F}^{\circ} \mathrm{F}^{\circ}$, aço e PRFV (Poliéster Reforça-

\footnotetext{
${ }^{2}$ Allievi é um software profissional para o cálculo e simulação de transitórios hidráulicos, e foi desenvolvido pelo Grupo de Engenharia e Tecnologia da Água da Universidade Politécnica de Valência. Utiliza como método de simulação o método das características.
} 
do com Fibra de Vidro), para as seguintes faixas: $0,7 \mathrm{~m} / \mathrm{s} \leq \mathrm{v} \leq 2,6 \mathrm{~m} / \mathrm{s}$ e 29 m.c.a $\leq H m \leq 95$ m.c.a

\subsection{Chaminé de Equilíbrio}

As chaminés de equilíbrio são muito utilizadas em sistemas hidrelétricos, pois há no geral favorecimento da topografia do terreno. Já em linhas de adução de água é economicamente difícil implantar a chaminé. Quando instalada adjacente às bombas, terá elevada eficácia, mas também elevada altura e volume, o que será oneroso e por muitas vezes inviável economicamente (Fig. 1a). Por outro lado, se instalada próxima ao reservatório superior (Fig. 1b), terá menor custo de implantação, mas perderá eficácia.
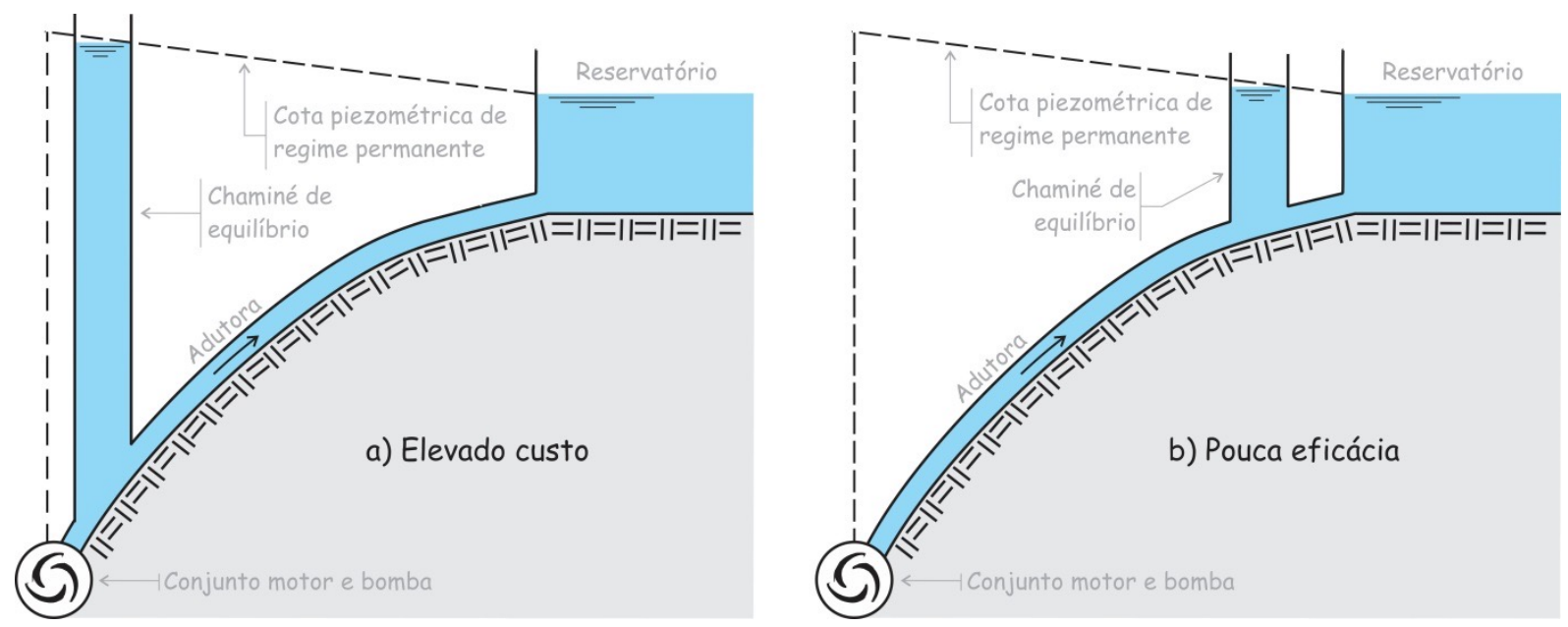

Figura 1 - Limites inviáveis de posicionamentos da chaminé de equilíbrio por ineficácia ou elevado custo.

Assim, a desvantagem da chaminé de equilíbrio está no fato de que, como o seu nível de água atinge a cota piezométrica de regime permanente no local, sua posição limita-se às cotas elevadas da linha de adução, para que seu volume/custo não seja excessivo. Por outro lado, não pode estar demasiadamente próxima ao reservatório superior, para que não perca sua eficácia. Neste estudo, foi adotada uma distância entre a chaminé e o reservatório de 4L/5.

De acordo com Eletrobras (1985), o primeiro procedimento é verificar a necessidade do uso de uma chaminé de equilíbrio no sistema hidráulico. O uso do dispositivo é necessário quando a Eq. 13 não for atendida.

$$
\frac{\mathrm{L}}{\mathrm{H}_{\mathrm{m}}} \leq 5
$$

Onde L é o comprimento total da linha de adução (em $\mathrm{m}$ ) e Hm é a altura manométrica (em m.c.a.). Nos casos em que o comprimento da adutora sobrepuser cinco vezes a $\mathrm{Hm}$, haverá a necessidade de prever uma chaminé de equilíbrio.

Eletrobras (1985) também cita o uso do critério da constante de desaceleração da água na tubulação, determinado pela Eq. 4.

Caso o valor de th for inferior a $3 \mathrm{~s}$, não haverá necessidade da chaminé de equilíbrio. Existem casos em que não se necessita de chaminé para valores até $6 \mathrm{~s}$ para th. Acima de $6 \mathrm{~s}$, a instalação da chaminé é obrigatória.

Para os casos em que a chaminé for obrigatória, são estudados os diversos tipos de dispositivo para ser implantado. Neste estudo foi utilizada a chaminé de equilíbrio do tipo simples e de seção constante (Fig. 2). 


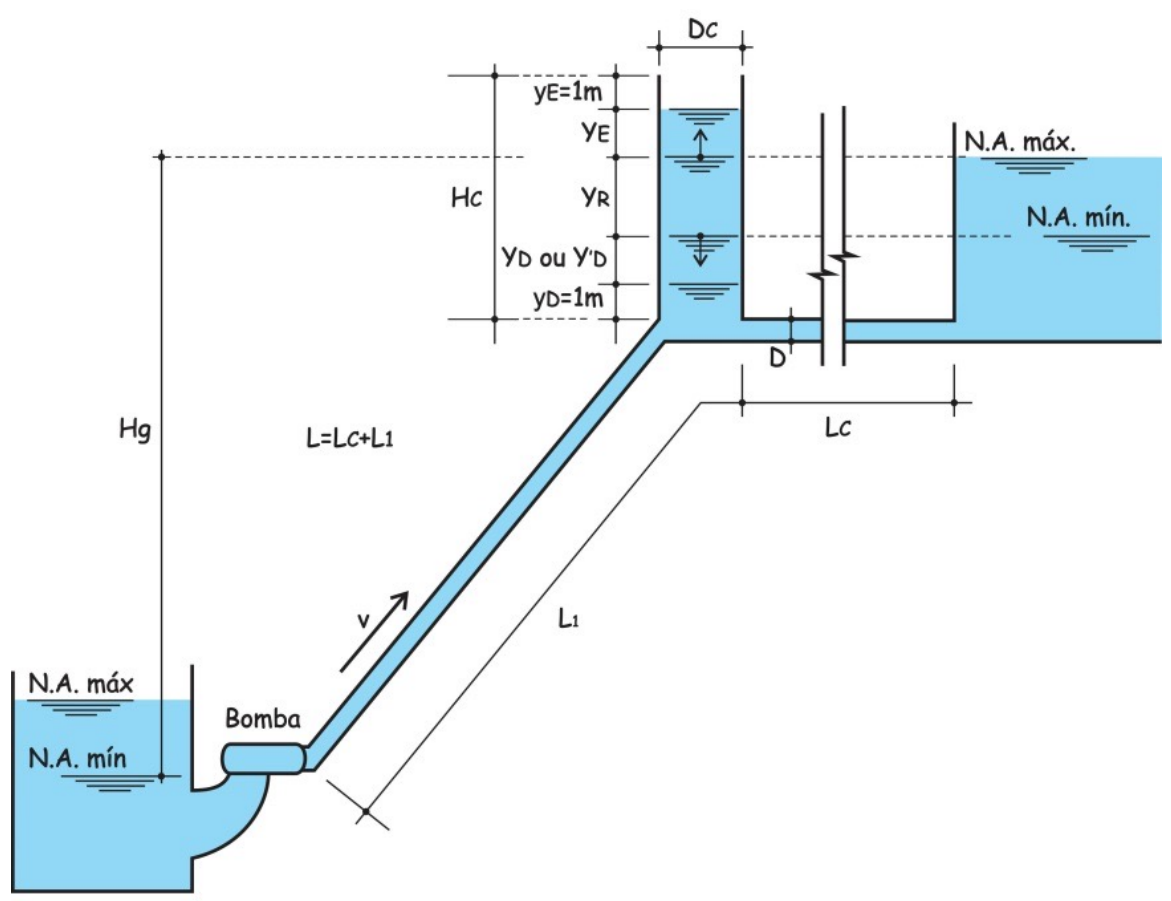

Figura 2 - Parâmetros utilizados do cálculo de uma chaminé de equilíbrio do tipo simples e seção constante. Fonte: adaptado de Eletrobras, 1985, p.215.

Para o cálculo desse tipo de chaminé, utiliza-se a Eq. 14, que permite determinar a área mínima da seção transversal desse dispositivo.

$$
A_{C} \geq \frac{v^{2}}{2 \cdot g} \cdot \frac{L_{C} \cdot A_{t}}{\left(H g-J_{c t}\right) \cdot J_{c t}}
$$

Onde $\mathrm{A}_{\mathrm{C}}$ é a área interna mínima da seção transversal da chaminé de equilíbrio $\left(\mathrm{em}^{2}{ }^{2}\right)$, vé a velocidade (em $\mathrm{m} / \mathrm{s}$ ) da água no interior da linha de adução, $L_{c}$ é o comprimento (em m) da linha de adução entre a chaminé e o reservatório de chegada da água (foi adotado no estudo, $L_{c}=4 L / 5$ ), At é a área interna da seção transversal da adutora entre o reservatório de chegada da água e a chaminé (em $\left.\mathrm{m}^{2}\right), \mathrm{Hg}$ é a altura geométrica do sistema (em $\mathrm{m}), \mathrm{J}_{\mathrm{ct}}$ é a perda de carga (em $\mathrm{m}$ ) total (distribuída - Jd + localizada - Jl) na adutora entre o reservatório de chegada da água e a chaminé e $\mathrm{g}$ é a aceleração da gravidade $\left(\mathrm{em} \mathrm{m} / \mathrm{s}^{2}\right)$.

A altura da chaminé de equilíbrio é determinada em função da oscilação do nível de água no seu interior. Tal oscilação pode ser calculada pela sequência a seguir.

Desprezando-se as perdas de carga $J_{\text {ct }}$ da linha de adução, pode-se determinar a elevação Ye ou depleção Yd do nível de água, em m, pela Eq. 15.

$Y_{e}=Y_{d}=v \cdot \sqrt{\frac{A_{t} \cdot L_{C}}{g \cdot A_{C}}}$

A elevação do nível de água da chaminé de equilíbrio é contada para cima do nível estático máximo e a depleção, para baixo do nível estático mínimo (ELETROBRAS, 1985).

Sendo consideradas as perdas de carga na linha de adução, tem-se:

$\mathrm{Y}_{\mathrm{E}}=\mathrm{z}_{\mathrm{e}} \cdot \mathrm{Y}_{\mathrm{e}}$

Em que:

$$
\mathrm{z}_{\mathrm{e}}=1-\frac{2}{3} \cdot \mathrm{J}_{\mathrm{R}}+\frac{1}{9} \cdot \mathrm{J}_{\mathrm{R}}^{2}
$$


Sendo:

$$
\mathrm{J}_{\mathrm{R}}=\frac{\mathrm{J}_{\mathrm{ct}}}{\mathrm{Y}_{\mathrm{e}}}=\frac{\mathrm{J}_{\mathrm{ct}}}{\mathrm{Y}_{\mathrm{d}}}
$$

Onde $\mathrm{Y}_{\mathrm{E}}$ é a elevação do nível de água, em $\mathrm{m}$ (considerando as perdas de carga), $J_{R}$ é a perda de carga relativa e $J_{c t}$ é a perda de carga total no sistema adutor entre a chaminé (em $\mathrm{m}$ ) e o reservatório de descarga da água, com a perda de carga Jd (perda de carga distribuída), por atrito, na tubulação adutora. $O$ cálculo da depleção $Y_{D}(e m$ $\mathrm{m})$, foi o resultante do fechamento total (100\%) do dispositivo de fechamento, sendo calculado pela Eq. 19.

$$
\mathrm{Y}_{\mathrm{D}}=\mathrm{z}_{\mathrm{d}} \cdot \mathrm{Y}_{\mathrm{d}}
$$

$O$ valor do coeficiente $z_{d}$ pode ser determinado pela Eq. 20, obtida por meio de uma curva polinomial ajustada a partir dos valores propostos por Eletrobras (1985, p.217).

$\mathrm{z}_{\mathrm{d}}=0,6535 . \mathrm{J}_{\mathrm{R}}^{2}-1,3066 . \mathrm{J}_{\mathrm{R}}+0,9625$

Para a segunda verificação, o procedimento é análogo ao da primeira:

$$
\mathrm{Y}_{\mathrm{D}}^{\prime}=\mathrm{z}_{\mathrm{d}}^{\prime} \cdot \mathrm{Y}_{\mathrm{d}}
$$

$O$ valor do coeficiente $z^{\prime}{ }_{d}$ pode ser determinado pela Eq. 22, obtida, assim como a Eq. 20, por meio de uma curva polinomial ajustada a partir dos valores propostos por Eletrobras (1985, p.219³).

$\mathrm{z}_{\mathrm{d}}^{\prime}=0,1735 \cdot \mathrm{J}_{\mathrm{R}}{ }^{2}-0,3165 \cdot \mathrm{J}_{\mathrm{R}}+0,5044$

A altura da chaminé de equilíbrio $(\mathrm{Hc})$ pode ser determinada conforme a seguir:

$$
\mathrm{H}_{\mathrm{C}}=\mathrm{Y}_{\mathrm{E}}+\mathrm{y}_{\mathrm{E}}+\left(\mathrm{Y}_{\mathrm{D}} \text { ou } \mathrm{Y}_{\mathrm{D}}^{\prime}\right)+\mathrm{y}_{\mathrm{D}}+\mathrm{Y}_{\mathrm{R}}
$$

Onde $y_{E}$ e $y_{D}$ são os acréscimos (segurança) nas alturas de elevação e da depleção (usa-se $1 \mathrm{~m}$ ), $Y_{R}$ é a depleção máxima do N.A. do reservatório de chegada de água, isto é, a diferença entre o N.A. máximo Normal e o N.A. Mínimo, em $\mathrm{m}$. Para o caso de SBAs em que o nível de água do reservatório de chegada não varie, adota-se $\mathrm{Y}_{\mathrm{R}} \approx 0$, pois a variação do N.A. do reservatório é desprezível. Por fim, o volume da chaminé pode ser determinado por:

$\mathrm{V}_{\mathrm{C}}=\mathrm{A}_{\mathrm{C}} \cdot \mathrm{H}_{\mathrm{C}}$

Onde $V_{c}$ é o volume da chaminé, em $\mathrm{m}^{3}, \mathrm{~A}_{\mathrm{C}}$ é a área da seção interna da chaminé, em $\mathrm{m}^{2}$, e $\mathrm{H}_{\mathrm{C}}$ é a altura da chaminé, em $\mathrm{m}$.

\section{METODOLOGIA}

O método de Tassinari (2017) foi aplicado aos 105 SBAs, conforme pode ser observado na Tabela 2.

\footnotetext{
${ }^{3}$ Os valores de $J_{R}$ expostos nesta pesquisa estão representados em Eletrobras (1985) de duas formas: como $J_{R}$ (para tubos lisos) e $J_{R}^{\prime}(p a r a$ tubos ásperos), pois o método de dimensionamento proposto é voltado para hidrelétricas. Nesta pesquisa foi realizada uma adaptação para sistemas de bombeamento, na qual as perdas de carga são calculadas pela fórmula de Hazen-Williams, embora também possam ser calculadas por Darcy-Weisbach. Assim, na adaptação deste dimensionamento de chaminé para sistemas de bombeamento, não foi estabelecida distinção entre tubos lisos e ásperos, logo, $J_{R}=J_{R}^{\prime}$.
} 
Tabela 2 - SBAs dentro e fora da faixa de aplicação do método de Tassinari (2017).

\begin{tabular}{|c|c|c|c|c|c|c|c|c|c|c|c|c|c|c|c|}
\hline SBA. & $\begin{array}{c}v \\
(\mathrm{~m} / \mathrm{s})\end{array}$ & $\begin{array}{l}\mathrm{Hm} \\
(\mathrm{m})\end{array}$ & $\begin{array}{c}\text { Está } \\
\text { na } \\
\text { faixa? }\end{array}$ & SBA. & $\begin{array}{c}v \\
(\mathrm{~m} / \mathrm{s})\end{array}$ & $\begin{array}{l}\mathrm{Hm} \\
(\mathrm{m})\end{array}$ & $\begin{array}{c}\text { Está } \\
\text { na } \\
\text { faixa? }\end{array}$ & SBA. & $\begin{array}{c}v \\
(\mathrm{~m} / \mathrm{s})\end{array}$ & $\begin{array}{l}\mathrm{Hm} \\
(\mathrm{m})\end{array}$ & $\begin{array}{c}\text { Está } \\
\text { na } \\
\text { faixa? }\end{array}$ & SBA. & $\begin{array}{c}v \\
(\mathrm{~m} / \mathrm{s})\end{array}$ & $\begin{array}{l}\mathrm{Hm} \\
(\mathrm{m})\end{array}$ & $\begin{array}{c}\text { Está } \\
\text { na } \\
\text { faixa? }\end{array}$ \\
\hline 1 & 2,42 & 11,80 & Não & 29 & 2,13 & 32,50 & $\operatorname{Sim}$ & 50 & 2,37 & 58,83 & $\mathrm{Sim}$ & 78 & 2,08 & 89,10 & $\operatorname{Sim}$ \\
\hline 2 & 1,67 & 10,74 & Não & 30 & 3,00 & 37,39 & Não & 51 & 2,16 & 56,34 & Sim & 79 & 2,02 & 86,47 & Sim \\
\hline 3 & 2,21 & 11,06 & Não & 31 & 1,22 & 26,86 & Não & 52 & 1,41 & 52,50 & Sim & 80 & 1,42 & 80,02 & $\operatorname{Sim}$ \\
\hline 4 & 1,26 & 10,35 & Não & 32 & 1,09 & 26,31 & Não & 53 & 1,04 & 51,14 & Sim & 81 & 2,58 & 87,74 & Sim \\
\hline 5 & 1,03 & 10,20 & Não & 33 & 2,26 & 29,49 & Sim & 54 & 1,30 & 51,57 & Sim & 82 & 0,86 & 76,37 & Sim \\
\hline 6 & 1,87 & 10,56 & Não & 34 & 2,53 & 29,86 & $\mathrm{Sim}$ & 55 & 2,93 & 56,18 & Não & 83 & 2,76 & 86,05 & Não \\
\hline 7 & 1,38 & 10,26 & Não & 35 & 0,50 & 25,19 & Não & 56 & 3,00 & 55,36 & Não & 84 & 3,00 & 86,23 & Não \\
\hline 8 & 2,06 & 16,59 & Não & 36 & 2,14 & 40,57 & Sim & 57 & 1,91 & 62,47 & Sim & 85 & 2,40 & 101,74 & Não \\
\hline 9 & 1,69 & 13,98 & Não & 37 & 1,53 & 31,92 & $\mathrm{Sim}$ & 58 & 2,31 & 64,78 & Sim & 86 & 2,35 & 101,52 & Não \\
\hline 10 & 1,19 & 11,78 & Não & 38 & 1,18 & 28,56 & Não & 59 & 2,49 & 62,93 & Sim & 87 & 1,88 & 100,80 & Não \\
\hline 11 & 1,61 & 12,70 & Não & 39 & 1,21 & 27,91 & Não & 60 & 2,08 & 58,50 & Sim & 88 & 2,45 & 101,19 & Não \\
\hline 12 & 2,80 & 16,62 & Não & 40 & 2,12 & 32,77 & $\operatorname{Sim}$ & 61 & 1,82 & 55,90 & Sim & 89 & 2,53 & 101,09 & Não \\
\hline 13 & 1,01 & 10,85 & Não & 41 & 0,68 & 25,81 & Não & 62 & 2,62 & 60,43 & Não & 90 & 2,70 & 101,09 & Não \\
\hline 14 & 0,54 & 10,23 & Não & 42 & 2,68 & 33,27 & Não & 63 & 2,18 & 56,06 & Sim & 91 & 1,21 & 100,20 & Não \\
\hline 15 & 2,38 & 27,45 & Não & 36 & 2,14 & 40,57 & $\operatorname{Sim}$ & 64 & 2,12 & 76,54 & Sim & 92 & 2,63 & 111,16 & Não \\
\hline 16 & 1,39 & 15,66 & Não & 37 & 1,53 & 31,92 & Sim & 65 & 2,31 & 76,32 & Sim & 93 & 2,11 & 106,25 & Não \\
\hline 17 & 1,28 & 14,12 & Não & 38 & 1,18 & 28,56 & Não & 66 & 2,62 & 76,41 & Não & 94 & 3,00 & 110,30 & Não \\
\hline 18 & 1,41 & 14,25 & Não & 39 & 1,21 & 27,91 & Não & 67 & 3,00 & 76,78 & Não & 95 & 2,78 & 107,51 & Não \\
\hline 19 & 2,75 & 22,72 & Não & 40 & 2,12 & 32,77 & $\operatorname{Sim}$ & 68 & 2,45 & 75,93 & Sim & 96 & 2,72 & 106,09 & Não \\
\hline 20 & 0,71 & 10,86 & Não & 41 & 0,68 & 25,81 & Não & 69 & 2,91 & 76,24 & Não & 97 & 2,49 & 104,44 & Não \\
\hline 21 & 0,88 & 11,02 & Não & 42 & 2,68 & 33,27 & Não & 70 & 2,78 & 75,85 & Não & 98 & 3,00 & 105,62 & Não \\
\hline 22 & 2,00 & 26,34 & Não & 43 & 1,97 & 51,33 & Sim & 71 & 2,47 & 84,92 & Sim & 99 & 2,09 & 114,83 & Não \\
\hline 23 & 1,84 & 25,93 & Não & 44 & 2,28 & 51,42 & $\operatorname{Sim}$ & 72 & 2,53 & 83,38 & Sim & 100 & 2,55 & 116,91 & Não \\
\hline 24 & 1,30 & 25,41 & Não & 45 & 1,90 & 50,81 & Sim & 73 & 1,11 & 76,58 & Sim & 101 & 1,52 & 105,31 & Não \\
\hline 25 & 0,85 & 25,16 & Não & 46 & 2,03 & 50,78 & $\operatorname{Sim}$ & 74 & 0,98 & 76,10 & Sim & 102 & 0,94 & 101,91 & Não \\
\hline 26 & 0,66 & 25,09 & Não & 47 & 1,15 & 50,26 & Sim & 75 & 3,00 & 83,19 & Não & 103 & 2,82 & 113,14 & Não \\
\hline 27 & 0,63 & 25,07 & Não & 48 & 2,88 & 51,25 & Não & 76 & 2,99 & 81,41 & Não & 104 & 3,00 & 113,77 & Não \\
\hline 28 & 1,42 & 25,26 & Não & 49 & 2,11 & 50,55 & Sim & 77 & 3,00 & 80,47 & Não & 105 & 2,46 & 107,56 & Não \\
\hline
\end{tabular}

Os SBAs analisados que estiveram na faixa de aplicação do método de Tassinari (2017) foram poucos, já que representaram apenas $33,33 \%$ de todos os casos. No entanto, essa característica não é parâmetro relevante para a análise da eficácia do método, dada a complexidade numérica transposta para criar o conjunto de equações analíticas que geram resultados explícitos.

$\mathrm{Na}$ Fig. 3 e na Fig. 4, são exibidas as envoltórias de pressões extremas geradas nos dois métodos discutidos nesta pesquisa. Para exemplificar isso, foram selecionados 12 SBAs que estão dentro da faixa de aplicação do método de Tassinari (2017). Esses SBAs têm $\mathrm{Hg}=50$ m e $\mathrm{Hg}=75 \mathrm{~m}$, todos os valores de $L(L=100 \mathrm{~m}, 500 \mathrm{~m}$ e $1000 \mathrm{~m})$ e foram comparados por seu menor ( $D i=276 \mathrm{~mm}$ ) e maior Di $(635 \mathrm{~mm})$. Em seguida foram plotadas as linhas piezométricas de regime permanente e os perfis fictícios de linhas de adução, para servir de referência ao traçado das envoltórias. 

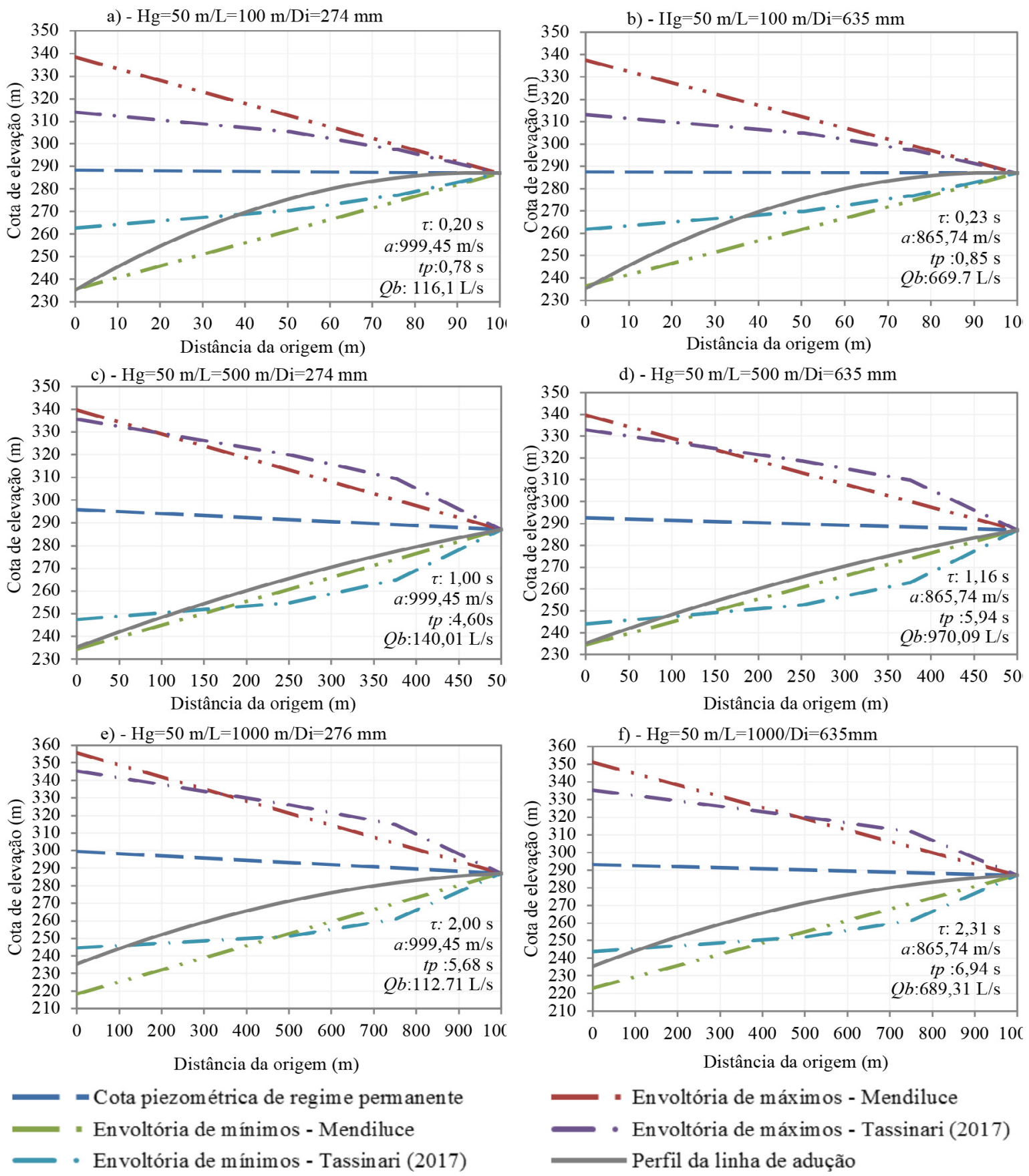

Figura 3 - Construção das envoltórias de pressões extremas pelo método de Mendiluce e método de Tassinari (2017) para SBAs com $\mathrm{Hg}=50 \mathrm{~m}$.

Inicialmente pode ser observado que as pressões extremas geradas pelo método de Tassinari (2017) são inferiores próximo à bomba, e que a elevação dos diâmetros não resultou em va- riações significativas nos valores das pressões extremas em nenhum dos métodos analisados. Também não foram verificadas variações nos menores comprimentos. 

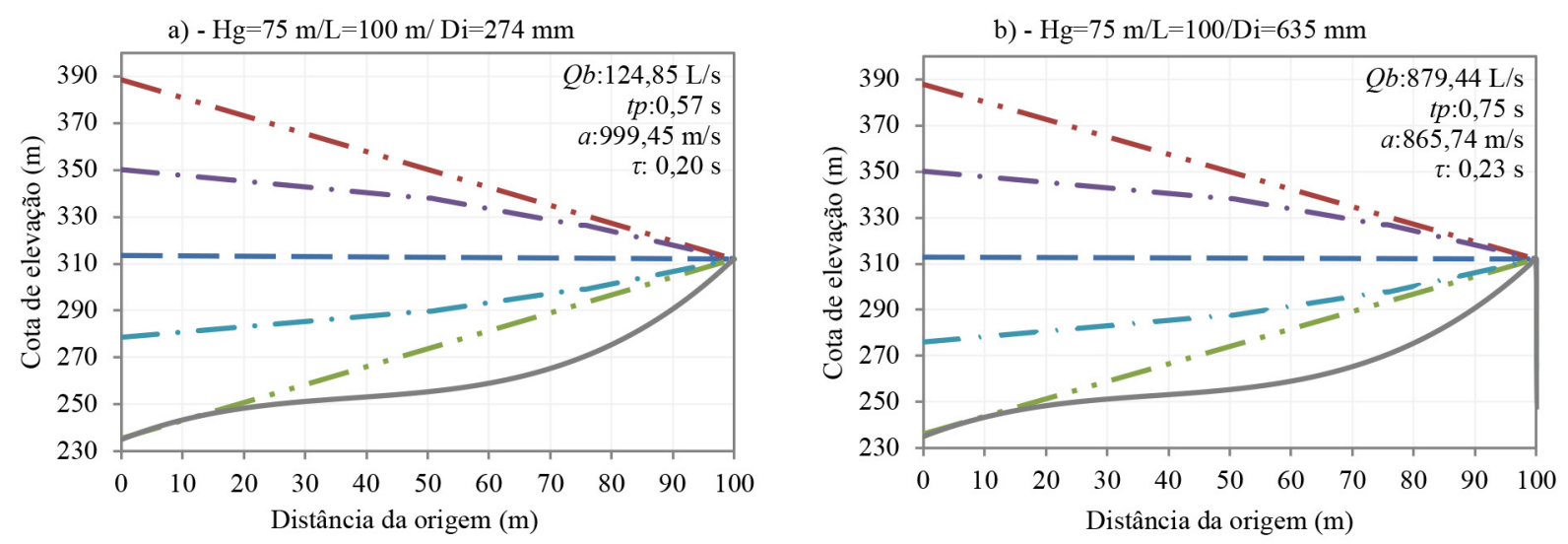

c) $-\mathrm{Hg}=75 \mathrm{~m} / \mathrm{L}=500 \mathrm{~m} / \mathrm{Di}=274 \mathrm{~mm}$
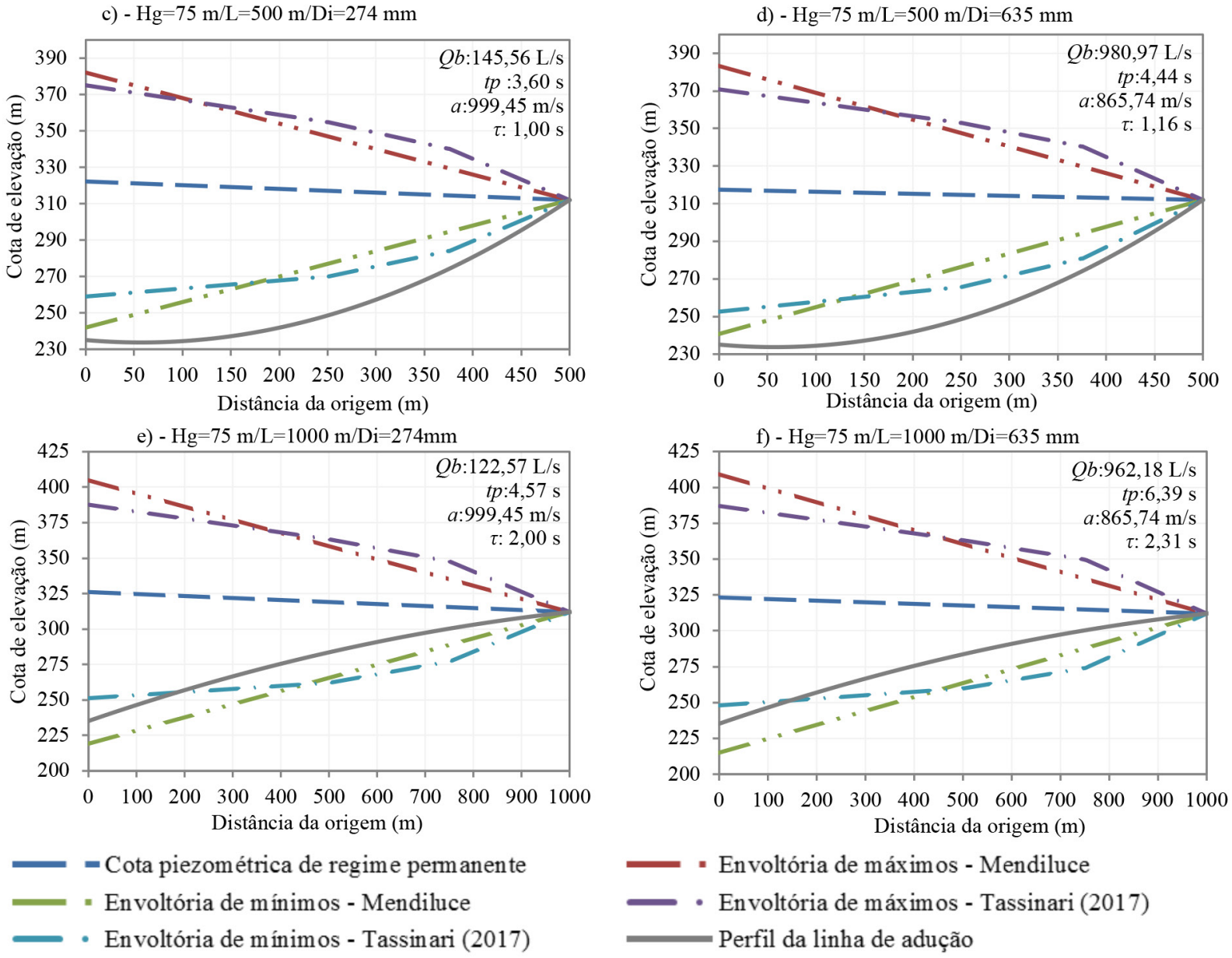

Figura 4 - Construção das envoltórias de pressões extremas pelo método de Mendiluce e método de Tassinari (2017) para combinações com $\mathrm{Hg}=75 \mathrm{~m}$.

Para os SBAs com L=100 m, as envoltórias de Tassinari (2017) não transpõem as envoltórias de Mendiluce em nenhum trecho das linhas de adução. Isso indica que o projetista do sistema de bombeamento deve optar pela segurança, empregando o método de Mendiluce para linhas de adução de até $100 \mathrm{~m}$.

Para as linhas com 500 m e 1000 m de comprimento, as envoltórias de Tassinari transpuse- 
ram em alguma parte do trecho as envoltórias de Mendiluce, resultando em maiores pressões extremas. Como exemplo, observa-se que as envoltórias de mínimos dos dois métodos ficaram abaixo da linha de adução na Fig. 3 (d). Nesse caso, a diferença entre as pressões extremas máximas e mínimas pelos dois métodos é da ordem de 10 m.c.a. nos 375 m (75\% do comprimento da linha) de distância da origem, indicando pressões mais extremas nas proximidades do reservatório para o método de Tassinari. É importante ressaltar que, em geral, tubulações de $\mathrm{F}^{\circ} \mathrm{F}^{\circ}$ apresentam boa resistência para pressões extremas máximas e mínimas, se comparadas, por exemplo, às tubulações de aço. No entanto, caso as pressões mínimas caiam a ponto de igualar a pressão de vapor da água para aquela temperatura de escoamento, pode ocorrer vaporização da água, formando cavidades de vapor que, devido ao gradiente de velocidade e inclinação do tubo, aderem à geratriz superior desse tubo. Esse fenômeno é denominado separação de coluna líquida (WANG et al., 2016), e é assim chamado devido ao fato de as cavidades atingirem dimensões equivalentes às da seção do escoamento pleno. Nesses casos, a força de inércia no fluxo transitório do tubo é a razão pela qual, após algum tempo, as cavidades de vapor colapsam, gerando picos repentinos de alta pressão. Geralmente essas cavidades crescem e colapsam repetidamente. Adamkowski e Lewandowski (2012) comentam que essas condições podem existir nas tubulações durante a rápida abertura / fechamento da válvula ou partida / parada das máquinas hidráulicas e podem causar a quebra dos sistemas hidráulicos ou até mesmo a destruição completa.

Assim, para linhas de adução entre aproximadamente 500 m e 1000 m, o método de Tassinari está a favor da segurança, sendo útil para o projetista antever e analisar pressões extremas (principalmente mínimas) adjacentes ao reservatório superior.
É importante lembrar que o modelo proposto por Tassinari (2017) foi elaborado com base nas interrupções comuns da operação de um CMB. Segundo o autor, apesar de citado que o transiente em um sistema de bombeamento é decorrente da parada brusca do bombeamento, na realidade, essa parada não é instantânea, já que no momento da interrupção do fornecimento de energia ainda existe um período de tempo até que a bomba pare efetivamente. Esse período de tempo depende do momento de inércia do conjunto elevatório.

Como o método de Tassinari (2017) foi desenvolvido considerando o momento de inércia do $C M B$ (parada lenta), os valores das pressões extremas de Mendiluce também deveriam apresentar as mesmas características. Portanto, o tempo de parada $(t p)$ utilizado para a determinação das envoltórias de Mendiluce foi calculado por meio da Eq. 3, que considera o coeficiente $K i$, que, por sua vez, representa o efeito da inércia das partes girantes do CMB. Por conta disso, todos os valores de $t p$ foram superiores ao período $\tau$ da tubulação. Isso significa que todas as paradas também são lentas no método de Mendiluce.

Como todo SBA é passível de transientes, foi preciso definir quais necessitam de chaminé de equilíbrio (ou outro dispositivo de proteção). Assim, foi realizada comparação entre o adimensional $\mathrm{L} / \mathrm{Hm}$ (Eq. 13) e o th (Eq. 4) para os 105 SBAs.

Foi observado que o adimensional L/Hm é favorável para a segurança do sistema, por indicar a necessidade de instalação de dispositivos de proteção no maior número de SBAs. Ainda é oportuno observar que não foram verificados SBAs em que o th indicasse a necessidade de dispositivo e o adimensional L/Hm não indicasse. No entanto, o inverso ocorreu.

Como L/Hm está a favor da segurança, não há necessidade de o projetista utilizar o th para a verificação de necessidade de dispositivo, já que L/ $\mathrm{Hm}$ cobre as possibilidades de th, além de abran- 
ger mais casos (ver Fig. 5). Assim, o adimensional L/Hm pode ser utilizado como principal método para o projetista verificar a necessidade de dispositivos de proteção contra transientes.

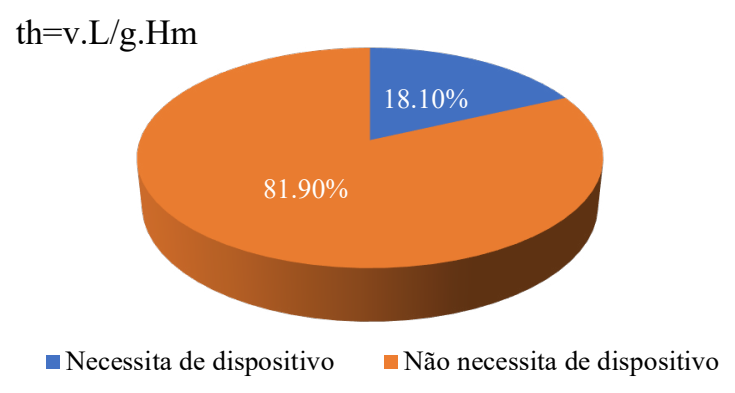

$\mathrm{L} / \mathrm{Hm}$

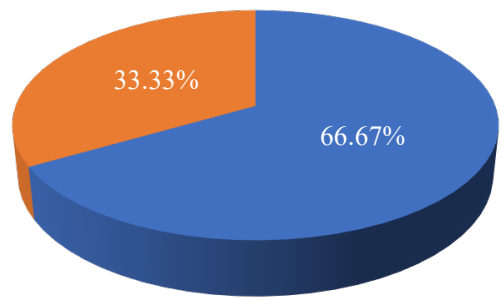

- Necessita de dispositivo

- Não necessita de dispositivo

Figura 5 - Percentual dos SBAs que necessitam de dispositivo de proteção pelo método do th e pelo adimensional L/Hm.

Após a determinação dos SBAs que requerem dispositivo de proteção, foi determinada a correlação entre Di e os parâmetros Q̣b, VRE e Vc (Fig. 6).
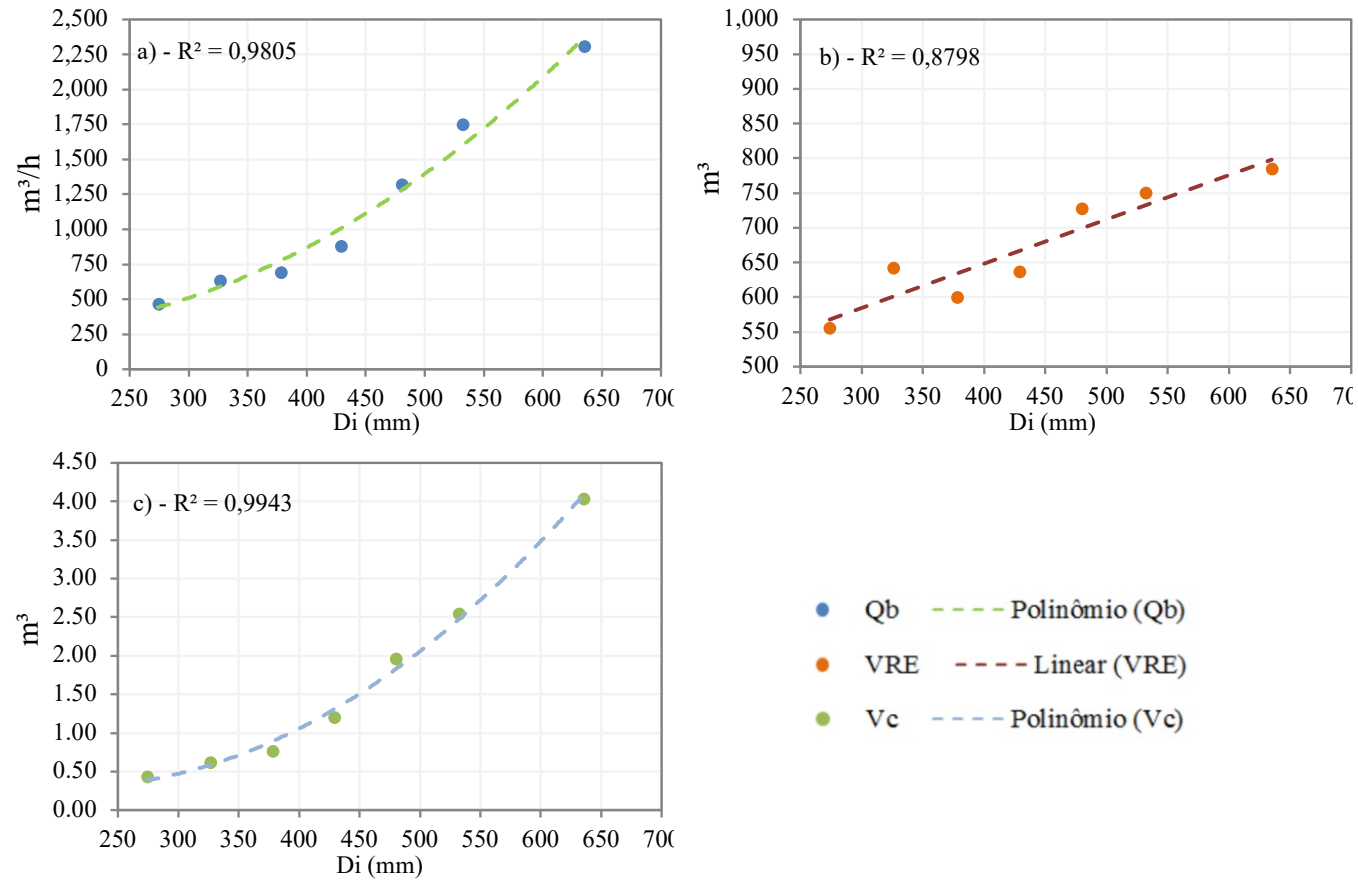

Figura 6 - Curvas ajustadas para a correlação entre Di e Q̣b, entre Di e VRE e entre Di e Vc para Lc=4L/5. 
As Eq. 25, 26 e 27 são resultantes das regressões da Fig. 6. Vale observar que houve elevado $\mathrm{R}^{2}$ para o ajuste exponencial dos dados da Fig. $6 \mathrm{a}$ e da Fig. 6c; no entanto, no ajuste polinomial, o valor foi maior.

$Q_{b}=0,0083 \cdot D i^{2}-2,1906 \cdot D i+420,57$

VRE $=0,6377 \cdot \mathrm{Di}+393,15$

$V_{c}=2 \cdot 10^{-5} \cdot D i^{2}-0,0088 \cdot D i+1,2201$

Onde Q Q é a vazão de bombeamento otimizada (em $\mathrm{m}^{3} / \mathrm{h}$ ), Di é o diâmetro interno da tubulação (em $\mathrm{mm}$ ), VRE é o volume útil do reservatório elevado (em $\mathrm{m}^{3}$ ) e Vc é o volume da chaminé de equilíbrio $\left(\mathrm{em} \mathrm{m}^{3}\right)$.

\section{CONCLUSÕES}

$\mathrm{Na}$ comparação dos métodos que geraram resultados para manobras lentas, foi observado que o método de Mendiluce está a favor da segurança nas proximidades do $\mathrm{CMB}$, já que as pressões extremas são maiores nessa posição.

O método de Tassinari (2017) tem as envoltórias de máximos e mínimos mais bem construídas, de modo que, para linhas de adução com comprimentos entre 500 m e 100 m, essas envoltórias transpõem as envoltórias de Mendiluce em algum trecho da linha, chegando a ser 10 m.c.a. mais intensas nas proximidades do reservatório superior (75\% de distância da origem). Com isso, o método de Tassinari está a favor da segurança nas adjacências do reservatório superior.

A recomendação destes autores é que, para estudos de concepção de sistemas de bombeamento com linhas de recalque em $\mathrm{F}^{\circ} \mathrm{F}^{\circ}$ de até $500 \mathrm{~m}$, seja utilizado apenas o método de Mendiluce. Para linhas de $\mathrm{F}^{\circ} \mathrm{F}^{\circ}$ a partir dos $500 \mathrm{~m}$ de comprimento, até $1000 \mathrm{~m}$, o método de Mendiluce pode ser utilizando em conjunto com o de Tassinari (2017), pois o primeiro é a favor da segurança próximo ao $C M B$, enquanto o segundo é a favor da segurança nas proximidades do reservatório superior. $O$ intervalo de $L$ entre $100 \mathrm{~m}$ e $500 \mathrm{~m}$ precisa ser estudado para o método de Tassinari (2017).

Quanto aos métodos de verificação de dispositivo de proteção, caso o projetista opte pela segurança, recomenda-se usar diretamente o adimensional L/Hm e estudar a posição eficaz mais econômica da chaminé de equilíbrio na linha, observando que o aumento do diâmetro da adutora poderá elevar o volume do dispositivo.

\section{AGRADECIMENTOS}

Os autores agradecem ao Conselho Nacional de Desenvolvimento Científico e Tecnológico (CNPq), à Coordenação de Aperfeiçoamento de Pessoal de Nível Superior (CAPES), ao Laboratório de Eficiência Energética e Hidráulica em Saneamento (LENHS) da UFRGS e ao Grupo Amazônico de Pesquisa em Saneamento Ambiental e Recursos Hídricos (SARH) da UFPA.

\section{CONTRIBUIÇÃO DOS AUTORES}

Conceitualização: LOPES, R. M, e MARQQUES, M.G.; Metodologia: LOPES, R. M, e MARQUUES, M.G.; Investigação: LOPES, R. M, e MARQQUES, M.G.; Redação - Primeira versão: LOPES, R. M.; MARQUUES, M.G.; DAI PRÁ, M. e PEREIRA, J. A. R Redação - Revisão \& Edição: LOPES, R. M.; MARQUES, M.G.; DAI PRÁ, M. e PEREIRA, J. A; Recursos: LOPES, R. M.; MARQUUES, M.G.; DAI PRÁ, M. e PEREIRA, J. A.

\section{REFERÊNCIAS}

ABNT. Associação Brasileira de Normas Técnicas. NBR 12215 - Projeto de adutora de água Parte 1: Conduto forçado. 1ed, 2017. 
ADAMKOWSKI, A.; LEWANDOWSKI, M. Investigation of Hydraulic Transients in a Pipeline with Column Separation. Journal of Hydraulic Engineering. v.138, n.11, p. 935-944, 2012. https://doi. org/10.1061/(ASCE)HY.1943-7900.0000596.

ELETROBRAS. Centrais Elétricas Brasileiras. Manual de mini-

centrais hidrelétricas. Rio de Janeiro: DNAEE, 1985. p 530.

GAD, A.A. M.; MOHAMMED, H. I. Impact of pipes networks simplification on water hammer phenomenon. Sadhana. v. 39, n.5, p. 1227-1244, 2014. https://doi.org/10.1007/s12046-014-0260-7.

KERAMAT, A. TIJSSELING, A.S.; HOU, Q.; AHMADI, A. Fluid-structure interaction with pipe-wall viscoelasticity during water hammer. Journal of Fluids and Structures. v. 28, n.12, p.434-455, 2012. https://doi.org/10.1016/j.jfluidstructs.2011.11.001.

LOPES, R. M.; MARQUUES, M.G.; TEIXEIRA, T.D.P.; PEREIRA, L.L.;SOUZA, D.E.S.2018 Estudo comparativo entre três métodos simplifi- cados de análise de transientes hidráulicos em linhas adutoras de água por bombeamento. In: XVI Simpósio Ítalo-Brasileiro de Engenharia Sanitária e Ambiental. Foz do Iguaçu, PR, 2018. Anais...

WANG, H.; ZHOU, L.; LIU, D.; KARNEY, B.;ASCE, M.; WANG, P.; XIA, L.; MA, j.; XU, C. CFD Approach for Column Separation in Water Pipelines. Journal of Hydraulic Engineering.v.142,n.10, p. 04016036-1 - 04016036-11, 2016. https://doi.org/10.1061/ (ASCE)HY.1943-7900.

TASSINARI, L.C.S. Transientes hidráulicos em sistemas de bombeamento: influência do material do conduto e dispositivo de proteção. Dissertação (Mestrado) - Instituto de Pesquisas Hidráulicas, UFRGS, Porto Alegre, 2017.

TRIKI, A. Water-hammer control in pressurized-pipe flow using an in-line polymeric short-section. Acta Mechanica. v. 227, n.3, p. 777-793, 2016. https://doi.org/10.1007/s00707-015-1493-1. 